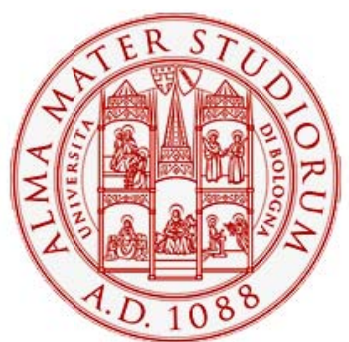

Alma Mater Studiorum - Università di Bologna DEPARTMENT OF ECONOMICS

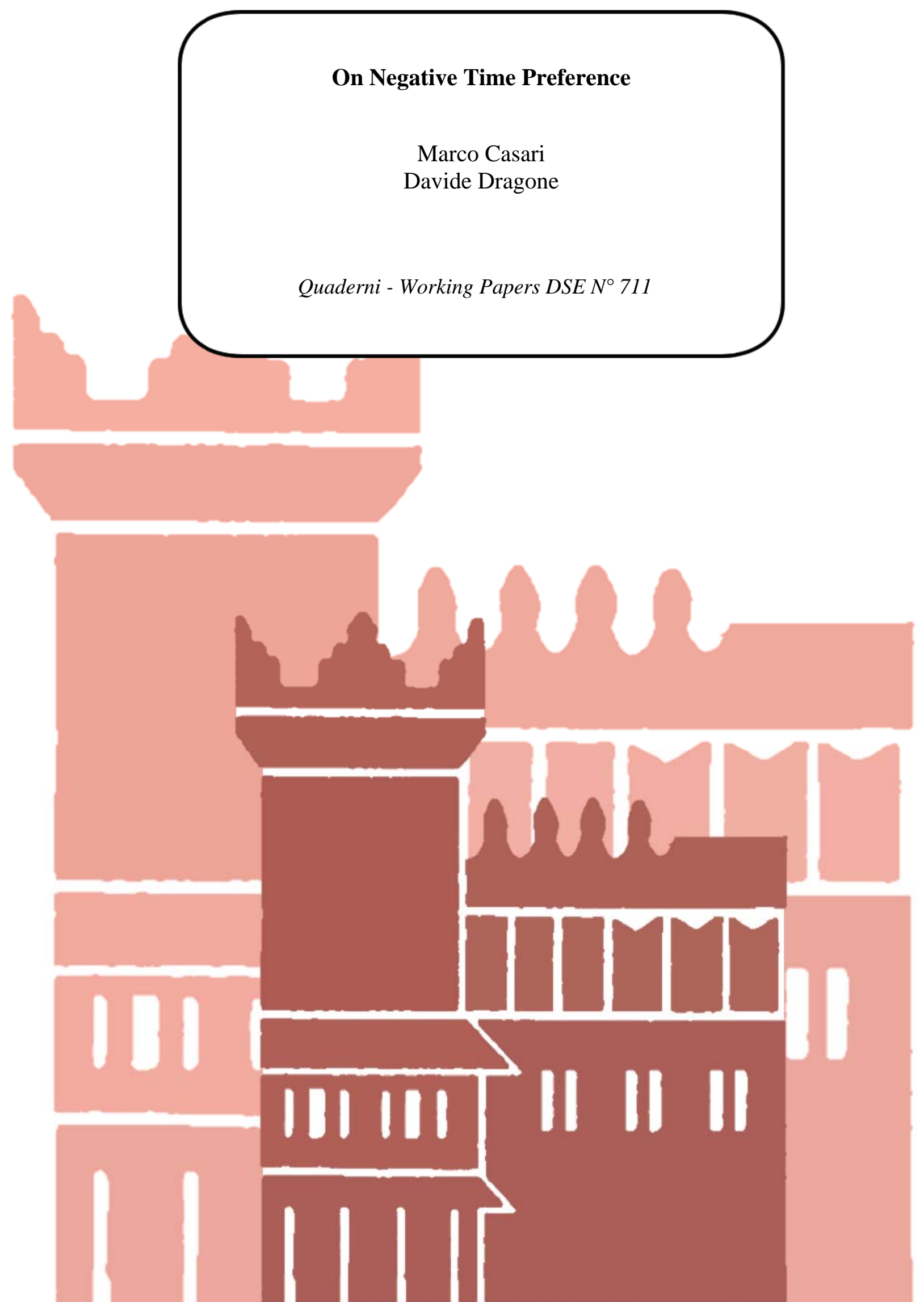




\title{
On Negative Time Preference
}

\author{
Marco Casari and Davide Dragone \\ University of Bologna
}

20 March 2010

\begin{abstract}
Survey data show that subjects positively discount both gains and losses but discount gains more heavily than losses. This holds for monetary and non-monetary outcomes.

JEL classification: C91, D90. Keywords: discounting, sequences
\end{abstract}

\section{Introduction}

Under positive discounting, a subject wants to anticipate a given reward and to postpone a given pain. In two well-known papers, Loewenstein (1987) and Loewenstein and Prelec (1991) question the general validity of the assumption that people (positively) discount future outcomes (Koopmans, 1960). ${ }^{1}$ They support this point by reporting intertemporal choices over hypothetical situations with monetary and non-monetary stimuli. While subjects positively discount money, they sometimes negatively discount non-monetary outcomes, such as a kiss from your favorite movie star or an electric shock (Loewenstein, 1987, L87 from now on). In addition, subjects greatly dislike sequences of dinners at restaurants that decline in value (Loewenstein and Prelec, 1991, LP91 from now on).

We conducted a similar survey. Our data show differences between discounting of gains and losses and little support for negative time preferences.

The paper is structured as follows. Section 1 describes the setup. Section 2 reports the results. Section 3 discusses the results within the literature and concludes.

\footnotetext{
${ }^{1}$ L87 has 223 citations and LP91 has 82 (18 March, 2010, Google Scholar, only published papers).
} 


\section{Design}

We asked to 190 undergraduates hypothetical questions about money equivalents and preferences over sequences. ${ }^{2}$

We asked four questions about money equivalents: (1) "What is the highest amount of money you are willing to pay now to receive with certainty 100 euros in the specified date?" Each subject stated an amount for five dates: "I am willing to pay at most euros now to receive 100 euros in 1 year" or in 4 weeks, or in 2 weeks, or 3 days, or now. ${ }^{3}$ The latter date was added as a control. The following questions had the same structure and concerned (2) "avoid losing with certainty 100 euros," (3) "avoid receiving with certainty a short, non-lethal 220-volt electric shock," (4) "receive with certainty a kiss from your favourite movie star/singer."

We asked three questions about preferences over sequences:

(5) "If we gave you a voucher for a dinner at the restaurant, which one of the following options would you prefer?" $(\mathrm{N}=186)$

- A dinner in a fish restaurant $(131,70.4 \%)$

- A dinner in a pizzeria $(55,29.6 \%)$

(6a) "If you answered that you would use the voucher in a fish restaurant, select your favourite option between the following ones:"

- A dinner in a fish restaurant on Saturday in one month $(112,85.5 \%)$

- A dinner in a fish restaurant on Saturday in two months $(19,14.5 \%)(\ldots)$

(7a) "select your favourite option between the following ones:"

- A dinner in a fish restaurant on Saturday in one month and a dinner in a pizzeria in two months $(91,69.5 \%)$

- A dinner in a pizzeria in one month and a dinner in a fish restaurant on Saturday in two months $(40,30.5 \%)$

(6b) "If you answered that you would use the voucher in a pizzeria, select your favourite option between the following ones:"

- A dinner in a pizzeria on Saturday in one month $(46,83.6 \%)$

- A dinner in a pizzeria on Saturday in two months $(9,16.4 \%)(\ldots)$

\footnotetext{
${ }^{2}$ University of Bologna, Italy; session dates: April $1^{\text {st }}$, May 13, 20, 22, 2009.

${ }^{3}$ Italics and bold in the original instructions.
} 
(7b) "select our favourite option between the following ones:"

- A dinner in a pizzeria on Saturday in one month and a dinner in a fish restaurant in two months $(48,87.3 \%)$

- A dinner in a fish restaurant in one month and a dinner in a pizzeria on Saturday in two months $(7,12.7 \%)$

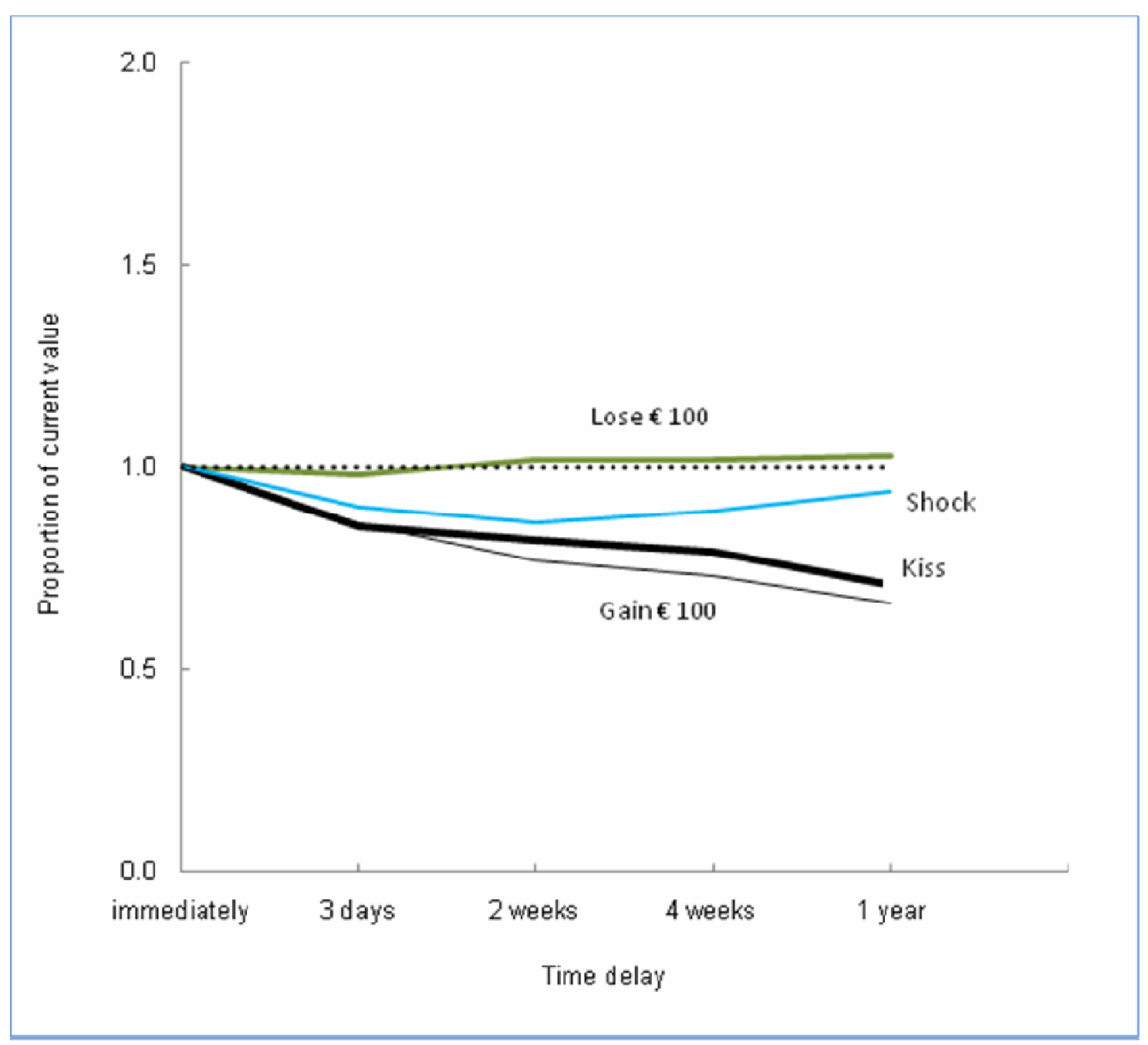

Figure 1: Maximum payment to obtain/avoid outcomes at selected time

Mean of the proportion of current value 
Notes: If on a single question a subject did not fill in all items, we dropped this observation. If on other questions the same subject filled in all items, we kept those observations. Hence, sample sizes may vary across questions. $N=178$ for 100 euro gain, 180 for 100 euro loss, 138 for kiss, 163 for shock.

\section{Results}

We report the results about the money equivalents questions (1-4) and then about the preferences over sequences questions (5-7).

On the money equivalent questions we find that subjects positively discount both gains and losses but discount gains more heavily than losses. First, subjects positively and monotonically discount future outcomes. Figures 1 illustrates the mean willingness to pay for outcomes at selected times, normalized by their current value ("nwtp"). By definition the lines originate at 1 because they are expressed as proportion of the current value. When a nwtp value is below 1 , it indicates positive discounting with respect to now. In Figure 1 all nwtp values, except loss of 100 euros, are always below 1. To support the result of positive discounting, we carried out four Wilcoxon matched-pairs signed-ranks tests for each question, and found that all nwtp values, including loss of 100 euros, are significantly below $1(\mathrm{p}<0.01, \mathrm{~N}=138-180$ depending on the question). A monotonic line in Figure 1 indicates monotonic discounting. All lines are monotonic, except the shock but this deviation is not significant. For each question we checked for significantly positive discounting in all pairs of nwtp values (3 days vs. 2 weeks, 3 days vs. 4 weeks, etc.). We carried out six additional Wilcoxon matched-pairs signed-ranks tests for each question and found that more distant values are always significantly lower $(\mathrm{p}<0.01, \mathrm{~N}=138-180$ depending on the question).

Second, subjects' intertemporal preferences are domain-specific. One can compare the nwtp values of different lines in Figure 1 for a given time delay. Sometimes, subjects discount differently kiss, shock, and monetary outcomes. For each time delay, we carried out six Wilcoxon matched-pairs signed-ranks tests and found that subjects discount the kiss differently than the shock at all time delays. Moreover, they discount the kiss differently than the 100 euro loss and they discount the shock differently than the 100 euro gain. ${ }^{5}$

\footnotetext{
${ }^{5} \mathrm{p}<0.05, \mathrm{~N}=123-174$ depending on the question. The kiss is not discounted differently than the 100 euro loss at 1 year delay $(\mathrm{p}=0.06)$ and the shock is not discounted differently than a 100 euro gain $(\mathrm{p}=0.06)$.
} 
The crucial feature of the stimulus is not whether it is monetary or non-monetary. The above tests reveal similar discounting of a 100 euro loss and a shock, and similar discounting of a 100 euro gain and a kiss. ${ }^{6}$ The relevant characteristic of the stimulus is whether it is in the gain or loss domain. Within monetary stimuli, a 100 euro gain is discounted differently than a 100 euro loss. Within non-monetary stimuli, a kiss is discounted differently than a shock. This distinction was also mentioned in LP91 and has been reported in the literature review of Frederick et al. (2002, p. 362-363).

On the preferences over sequences questions we report, first, that an overwhelming majority of subjects positively discount dinner outcomes; and, second, that this tendency is stronger for individual items than for sequences. When comparing individual choices in questions 5 and 6 , only $15.0 \%$ of subjects prefer to delay their best option, i.e. exhibit negative time preferences on single-outcome events $(n=28)$. When comparing sequences of events, more subjects prefer to delay their best option. More precisely, 25.3\% of individual choices in questions 5 and 7 reveal negative time preferences $(n=47)$. Finally, when checking for consistency in choices across questions 5, 6, and 7, about $69.9 \%$ always exhibit positive time preferences, and $10.2 \%$ always negative time preferences.

\section{Discussion}

Some of our results are different from those in the literature. Figure 2 reports the results of L87, which are similar to ours for the monetary outcomes and different for the non-monetary items. In Figure 2 subjects sometimes negatively discount a kiss and a shock, which we did not find.

\footnotetext{
${ }^{6}$ The 100 euro loss and shock are discounted differently at 2 weeks; the 100 euro gain and the kiss are discounted differently at 1 year (5\% significance level).
} 


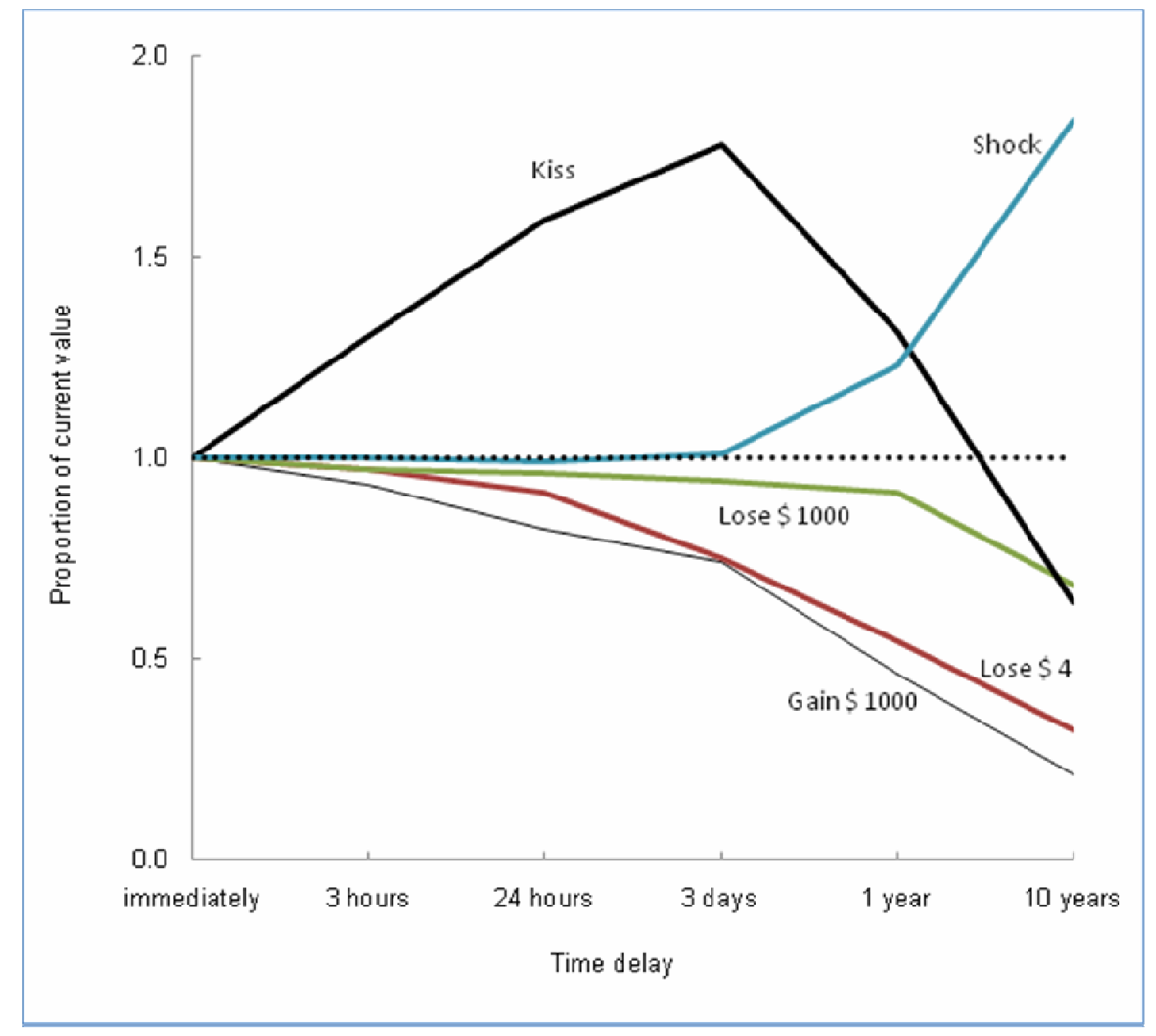

Figure 2: Maximum payment to obtain/avoid outcomes at selected time in Loewenstein (1987)

Mean of the proportion of current value

LP91 state that outcomes that decline in value are greatly disliked and report the supporting evidence that: "Of the 86 percent of subjects who preferred the fancy French dinner, 80 percent preferred a more immediate dinner over a more delayed dinner. However, when the French dinner was composed into a sequence with the Greek dinner, a slight majority (57 percent) preferred to have the better dinner come later. Even with single-outcome events there is some motivation to defer the French dinner -witness the 20 percent of subjects who opted for the longer delay. “ (p. 348). 
Based on questions 5-7 of this study, of the $70.4 \%$ of subjects who preferred the fish dinner, 85.5\% preferred a more immediate dinner over a more delayed dinner. However, when the fish dinner was composed into a sequence with the pizza dinner, a minority $(30.5 \%)$ preferred to have the better dinner come later. With single-outcome events there is some motivation to defer the fish dinner - as $14.5 \%$ of subjects opted for the longer delay. To conclude, subjects compatible with negative time preferences exists but in our study are a smaller fraction of about $10-20 \%$ of the sample.

One can put forward various conjectures for these discrepancies. One is that hypothetical questions generally yield noisy responses. To take care of it, we increased sample size and also looked at median instead of mean answers. While L87 involved 30 US undergraduates and LP91 involved 95 Harvard undergraduates, our sample size is 190. When checking results in Figure 1 in terms of median, they confirm positive time discounting. Another conjecture may be subject pool differences. L87 and LP91 conducted their study in the US while this study was conducted in Italy. Finally, there were some procedural and wording differences. Some of the time delays for the money equivalent questions were different from L87: we dropped 3 hours, 24 hours, and 10 years; we added 2 weeks and 4 weeks. L87 employed two money amounts (\$4, \$1000) while we employed just one $(100 €)$. Restaurant types were adjusted to fit the Italian culture. LP91 dinners were at either a fancy French or a local Greek restaurant. Frederick and Loewenstein (2008) found that procedural issues influenced results.

L87 and LP91 employed a survey as an illustrative example for a model where utility can depend not only on the consumption of a good (or a bad), but also on the anticipation of consumption. This argument sounds intuitive and appealing and should not be discarded simply because our data do not find support for it. Yet, the role of the experimenter is to try to replicate these studies. In the about 20-years period between the original experiments and our study, we have not found a published replication of this important result. Our results suggest that the behavioral difference in discounting is less between monetary and non-monetary stimuli, and more between gains and losses. 


\section{Bibliography}

Frederick, S. and Loewenstein, G., 2008, Conflicting motives in evaluations of sequences, Journal of Risk and Uncertainty, 37, 221-235

Frederick, S., Loewenstein, G., and O'Donoghue, T., 2002, Time discounting and time preference: A Critical Review, Journal of Economic Literature, 40(2), 351-401

Koopmans T. C., 1960, Stationary Ordinal Utility and Impatience, Econometrica, 28(2), 287309.

Loewenstein, G., 1987, Anticipation and the Valuation of Delayed Consumption, Economic Journal, 97(387), 666-84.

Lowenstein, G., and Prelec, D., 1991, Negative Time Preference, American Economic Review, $81(2), 347-52$. 


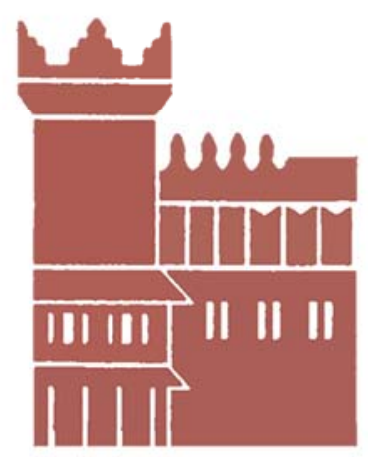

Alma Mater Studiorum - Università di Bologna DEPARTMENT OF ECONOMICS

Strada Maggiore 45

40125 Bologna - Italy

Tel. +39051 2092604

Fax +390512092664

http://www.dse.unibo.it 